Discussion Papers in Economics

\title{
Effects of Information on Environmental Quality in Developing Countries
}

\author{
E. Somanathan
}

June 2010

Discussion Paper 10-04

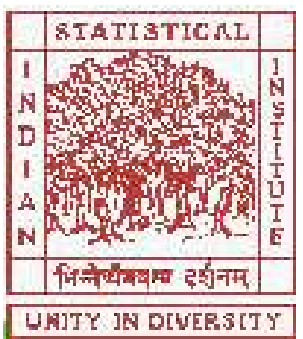

Indian Statistical Institute, Delhi

Planning Unit

7, S. J. S. Sansanwal Marg, New Delhi 110016, India 


\title{
Effects of Information on Environmental Quality in Developing Countries ${ }^{1}$
}

\author{
E. Somanathan ${ }^{2}$
}

Abstract: How does information on environmental risks obtained by individuals in developing countries affect environmental quality? The literature reveals that for issues like water quality and pesticides, information affects individual behavior and risks are reduced through individual action. However, even if information were to become widely available in developing countries, unless regulation is also strengthened, environmental risks will remain at high levels relative to developed countries. While education appears to raise the demand for environmental quality, there is no systematic developing-country evidence that this demand translates into increased supply through the political process and government regulation.

JEL classification numbers: I18, O13, Q51, Q56.

Key Words: Information, environmental quality, developing countries, valuation by revealed preference.

\section{Introduction}

We should expect low-income countries to have a lower demand for environmental quality than high-income countries. Since consumption (of other goods) is low in developing countries, there will be less willingness to sacrifice it for the sake of a cleaner environment. This argument helps to explain the low levels of environmental regulation in developing countries. Moreover, it suggests that such low levels of regulation may be socially optimal. Of course, there are other explanations for the weakness of environmental regulations in developing countries. For example, the political process in poor countries may produce sub-optimally low regulation, and one of the reasons for this could be low levels of public awareness about the environment. If people are not aware of environmental risks, they will be less willing to pay to reduce them. Thus, providing people with information about environmental hazards may increase welfare by raising environmental quality to a more appropriate level.

This argument about the link between public awareness (or information) and environmental quality assumes that the public's existing beliefs about environmental risks are biased downward. However, even if this were not the case, better information would still

\footnotetext{
${ }^{1}$ I am grateful to Subhrendu Pattanayak for pointing me to some of the literature, and to Jeff Vincent, Suzy Leonard, and an anonymous referee for helpful comments.

${ }^{2}$ Indian Statistical Institute, Delhi. E-mail: som@isid.ac.in
} 
increase welfare because it would allow pollution control to be adjusted upward or downward as appropriate. But the conclusion that environmental quality will improve with better information depends on the assumption of downwardly biased beliefs. How plausible is this assumption? It depends on how much is already known about the environmental risk in question. If people know that particulate matter suspended in the air is hazardous, but not how hazardous, then it is not clear whether the risk will be over-estimated or under-estimated. However, if they do not know that particulate matter is hazardous at all, they are very likely to act as if the risk is negligible. ${ }^{3}$ This is entirely natural. Lots of things could conceivably be dangerous, so life would be paralyzed if we were to assign positive probabilities to all imaginable risks.

Why should we expect information about environmental risks to be less available in lowincome countries? First, education levels are lower, so even if educated elites are well-informed, the majority of those exposed to risks may not be. For example, even though the germ theory of disease was developed more than a century ago, many people in developing countries are still unaware of it (Quick et al 1997, Jalan and Somanathan 2008). Second, because information about environmental risks is a public good, and its analysis often requires specialized training and expensive data collection, it will be under-supplied by individual action. This under-supply will tend to be more acute in countries in which education and data-processing capabilities are scarce. There is, therefore, good reason to expect that information about environmental quality will be under-supplied in poor countries in the absence of public action. Just how important information is, and how big a role it can play in alleviating environmental problems in developing countries, is of course an empirical matter.

The discussion thus far has implicitly assumed that information about environmental risks changes outcomes via government regulatory action in response to public demand. However, when it is feasible for individuals and households to take action to reduce their exposure to an environmental hazard, changes in outcomes can also come about through direct action by households. Two major environmental problems in low-income countries -- lack of

\footnotetext{
${ }^{3}$ Ironically enough, in pointing out that pollution in low-income countries is more acceptable than in high-income countries, Lawrence Summers illustrated his argument by referring to "visibility-impairing particulates ... [that] may have very little direct health impact", thus inadvertently highlighting the role of missing information. At the time, knowledge of the adverse health impacts of particles was far less widespread than it is today (Anonymous, 1992).
} 
safe water, sanitation and hygiene, and indoor smoke from solid fuels -- can be addressed at the household level, if only partially. In fact, these are the two environmental risk factors that made it onto the World Health Organization's list of 10 leading risk factors in high-mortality developing countries (WHO 2002). Another problem that can be partially addressed through individual action is pollution from pesticides.

The purpose of this article, which is part of a symposium on Environmental Quality and Economic Development, ${ }^{4}$ is to assess how information on environmental risks affects environmental quality in developing countries, through changes in individual behavior as well as government regulation. The next section briefly introduces some key concepts and an analytical framework for examining the causal links between information about environmental risks, individual and government actions, and environmental outcomes. This is followed by reviews of the empirical evidence on the role that information has played in household or individual actions to reduce water pollution and pesticide use. ${ }^{5}$ Next I discuss the effects of public awareness on government actions and environmental outcomes. The final section identifies and discusses the lessons from this review.

\section{Key Concepts and Analytical Framework}

Figure 1 provides a simple illustration of the possible causal links between information, government and individual actions, and environmental outcomes. Information and public awareness can raise the demand for environmental quality. This, in turn, may lead to private and/or government actions that affect environmental outcomes. Government actions such as monitoring pollutants and publishing information about them may also increase environmental

\footnotetext{
${ }^{4}$ The other articles in the symposium include Vincent (2010), which provides an overview of the issues and an introduction to the symposium; Blackman (2010), which discusses experience with alternative pollution control policies in developing countries; and Pattanayak, Wunder, and Ferraro (2010), which examines the empirical literature on payments for environmental services in developing countries.

${ }^{5}$ Unlike waterborne disease, which has been well-understood for more than a century, the dangers of indoor air pollution from solid fuel use for cooking have only been established over the last two or three decades (see e.g., Smith (2000) and Ezzati and Kammen (2002)). Because much less has been done to address this issue, and informational interventions in particular have not been attempted or studied, indoor air pollution is not addressed in detail in this article.
} 
information available to the public. Of course, the public may acquire information through other channels, for example, simply because the environment degrades noticeably.

Figure 1: Possible causal links between information, actions, and outcomes.

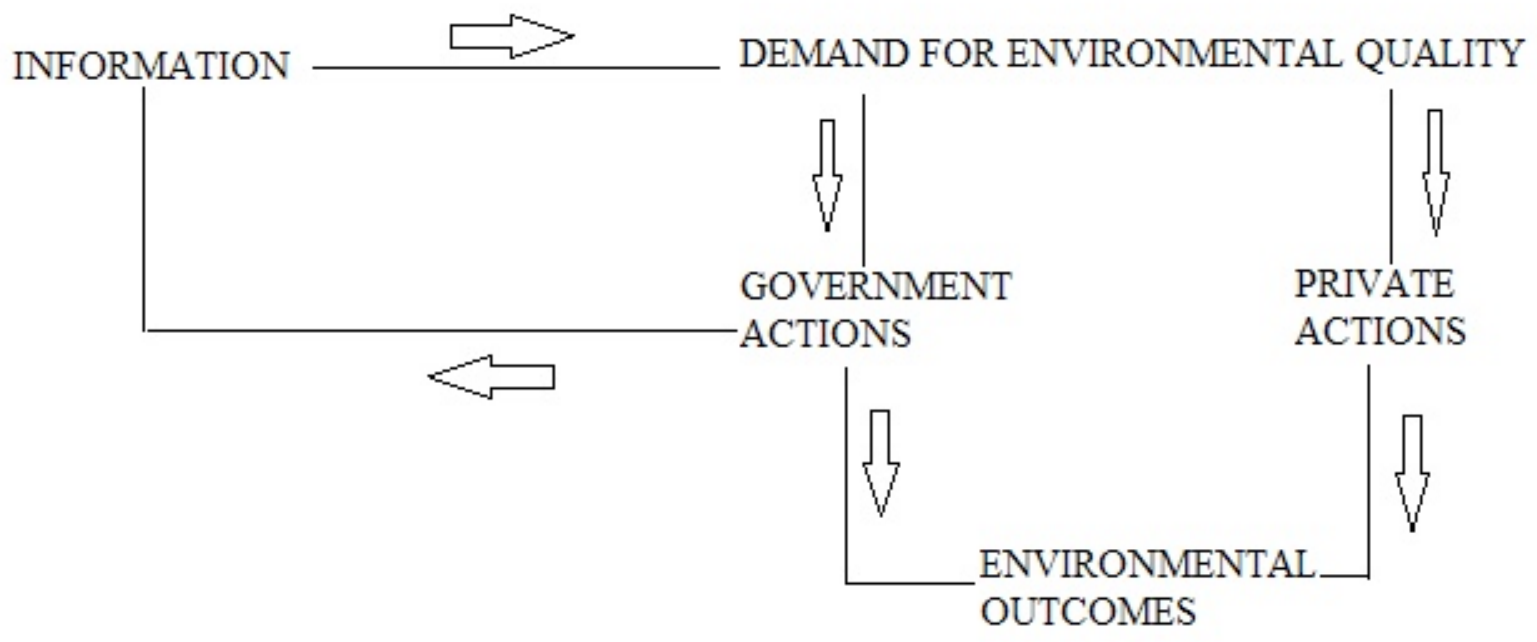

Figure 1 over-simplifies the way in which information can affect private actions in at least one important respect. This concerns the way in which people change their behavior in response to new information. Humans do not necessarily react to new information by rationally examining its implications. They are also heavily influenced by what their neighbors do without necessarily understanding the reasons for their neighbors' actions. While this behavior may not be individually rational in many situations, there is nevertheless a good reason why it is ubiquitous.

In their classic book, Culture and the Evolutionary Process (1985), Rob Boyd and Peter Richerson analyze imitation and individual learning. They ask: Under what circumstances would natural selection acting on genes favor imitation over learning from one's own experience as a learning strategy? In their framework, individuals face the problem of finding the best level of some behavior $X . X$ could represent, for example, the amount of time spent gathering easily available low-calorie plant foods rather than hunting. Individuals learn from their own personal experience and experimentation what behavior is appropriate to their habitat. Suppose such 
individual learning suggests that the optimal level of $X$ is $I$. Individuals also observe average behavior (the "culture") in the previous generation, $C$. They then adopt a weighted average of $C$ (cultural transmission) and $I$ (individual learning). The reliance on culture $C$ reduces sampling error relative to $I$ but may be inappropriate because the habitat may change between generations. Boyd and Richerson (1985) show that when there is some, but not too much autocorrelation in the habitat between generations, then natural selection gives a positive weight to both $C$ and $I$. If there is very little autocorrelation in the habitat, then the weight given to culture is zero. On the other hand, if autocorrelation is close to one, so that the habitat is very persistent, then there would be no role for learning of any kind and behavior would be hard-wired by genetic selection. Since humans evolved in a fluctuating environment in which ice ages and interglacials alternated but with autocorrelation on a generational timescale, it is plausible that we are genetically hardwired to rely both on cultural transmission and individual learning.

What implications does this have for behavioral responses to new information about environmental health and safety? The immediate implication is that households will give only partial weight to new information, with behavior learned from neighbors and others continuing to play an important role. A second implication is that adoption of a new behavior in a community may be a self-reinforcing process. The policy implication of this is that a "big push" strategy to take advantage of increasing returns may be called for.

\section{Information, Individual Actions, and Water Quality}

This section discusses the empirical evidence on the impact of information on water quality. First, a brief overview of the historical evidence about mortality and public health campaigns in developed countries, particularly the United States, is presented. This is followed by a detailed discussion of the evidence from developing countries concerning the impacts of hygiene education, formal schooling, individuals' occupations, and the news media on water quality and households' willingness to pay for safer water.

\section{Evidence from Developed Countries}


Historical evidence from now developed low-mortality countries at a time when their mortality rates were higher than those in high-mortality countries today strongly suggests that information, education and awareness campaigns improved hygiene and reduced mortality. Samuel Preston studied the early $20^{\text {th }}$-century United States (Preston 1996). In 1900, life expectancy in the United States was 48 years, much lower than, for example, India's life expectancy of 60 years in 2000. Life expectancy in the US increased rapidly during the twentieth century, to 78 years. This was not due to curative medicine, as the bulk of the declines in infectious diseases came before effective medical treatment became available. Nor was it due to improved nutrition. The US in 1900 was already a well-fed country with calorie consumption per equivalent adult higher than it was a hundred years later. The main reason for the increase in life expectancy was the improved disease environment, resulting from a number of factors: improved sanitation, a cleaner water supply (Cutler and Miller 2005), immunization, and improved hygiene. ${ }^{6}$

The early part of the century saw major public health campaigns aimed at reducing disease transmission by getting people to boil bottles and milk, wash hands, protect food from flies, isolate sick children, and so on (Preston 1996). New York City's public health department established milk depots whose real purpose was to instruct indigent mothers in hygienic practices. It also produced one of the first movies ever made, called The Fly Pest. The national Children's Bureau's pamphlet on infant care was the largest selling government publication at that point in history. Ewbank and Preston (1990) examined the impact of these campaigns by comparing the mortality of professional workers' children relative to the average before and after the campaigns were underway. Owing to their education, professionals such as teachers and doctors were more likely to be exposed to the messages and to understand their implications. They found that the relative mortality of professionals' children fell from $92 \%$ of the population mean in 1905 to $70 \%$ in $1922-24$. The relative declines for physicians and teachers over this period were even steeper, $92 \%$ to $65 \%$ and $104 \%$ to $61 \%$ respectively. These relative declines could not be accounted for by changing relative incomes or urbanization.

\footnotetext{
${ }^{6}$ Throughout this article, the term "sanitation" is used in its narrow public health sense to refer to the safe disposal of sewage and waste, while "hygiene" refers to behavior that reduces the transmission of infectious diseases.
} 


\section{Impact of Hygiene Education in Developing Countries}

How do public education efforts affect the disease environment in developing countries today? The easiest way that households can reduce their risk of water-borne and other infectious diseases is by improving hygiene since this does not entail investment in equipment. There are now many studies examining the causal link between hygiene and infections (Aiello and Larson 2002). The review by Aiello and Larson concluded that there is a "continued, measurable, positive effect of personal and community hygiene on infections". Both observational studies and interventions were reviewed. The 30 interventions reviewed consisted mainly of hygiene education (23 studies) and handwashing practices (6 studies). 15 of these interventions were in less-developed countries and were mainly within the community or families, unlike the developed-country studies that were in schools or day-care centers. Two of the thirty studies found no statistically significant effect of an educational intervention on disease, while the rest found appreciable reductions in the risk of disease, generally by more than $20 \%$, and in some cases by more than $70 \%$. Since a randomized design was difficult in many cases, only three of the studies used randomized designs. Two of these three found significant reductions in the risk of diarrhea, of $26 \%$ and $11 \%$ in Bangladesh and Zaire respectively, and one (in Burma) did not.

In a meta-analysis of the link between hygiene and infections, Fewtrell, Kaufmann et al. (2005) pooled the data from 11 hygiene interventions in less developed countries. The interventions consisted of hygiene education or promotion of handwashing in conjunction with education. Fewtrell, Kaufmann et al. (2005) found that the relative risk of diarrhea or dysentery was reduced by $37 \%$ and $45 \%$, respectively. ${ }^{7}$

Fewtrell et al.'s review also studied the effects of interventions that combined hygiene education with water provision and/or sanitation. Surprisingly, there was no evidence that combined interventions resulted in greater morbidity declines than those that intervened only in water supply, sanitation, or hygiene. It is also remarkable that the meta-analysis of interventions in water supply, water quality and sanitation came up with relative risk reductions no greater than those for hygiene education or hygiene education and handwashing. It should be noted, however, that there is some evidence of publication bias in the studies of hygiene and water

\footnotetext{
${ }^{7}$ The estimates for dysentery excluded two studies deemed to be of poor quality.
} 
supply, though not those of sanitation (Fewtrell et al 2005). ${ }^{8}$ This could mean that the results of the meta-analysis overstate the benefits of hygiene and water supply interventions.

The review did not provide information on the costs of the various interventions. However, these costs may not be an appropriate measure of the costs of undertaking large-scale interventions. It would be cheaper to provide the information on hygiene as part of primary education and through the news media. Hygiene information targeted at a small group is useful to identify an effect, but not for implementing policy. It seems very likely that information on hygiene as part of a broader education would be a cost-effective intervention.

\section{$\underline{\text { Impact of General Education }}$}

While the public health literature cited above examines the effect of specific information about hygiene on the disease environment in developing countries, there is also evidence that education in general is associated with better health. Several developed-country studies have found that schooling is associated with better health, controlling for correlates like income (Grossman 1976; Farrell and Fuchs 1982; Kenkel 1991). In a study of piped water provision from a nationally representative survey of rural India (Jalan and Ravallion 2003), ${ }^{9}$ children in households with piped water were found to be at lower risk of diarrhea, but this effect was found only for better-off households and in those in which the mothers had some schooling.

There is evidence that schooling leads people to adopt risk-reducing behavior, specifically in relation to water quality. Higher education levels were associated with a higher probability of home water purification in a survey of slum households in Delhi, India, controlling for income and other characteristics (Dasgupta 2004). This was found to be the case in urban Brazil as well (McConnell and Rosado 2000). In studies conducted by the World Bank in the

\footnotetext{
${ }^{8}$ Publication bias refers to the possibility that articles that find statistically significant effects may be more likely to be published than those that do not. Thus the selection of (published) articles in a meta-analysis could be biased towards a positive finding. A test for this phenomenon is based on the idea that a scatterplot of precision against estimated effect size will have an inverted funnel shape if there is no bias, but an asymmetric funnel with the left side cut off if there is publication bias (see Begg and Mazumdar 1994). There were fewer sanitation studies and this could explain the lack of evidence for publication bias in this case.

${ }^{9}$ The study compared children in households with piped water with children in otherwise similar households that did not have piped water using a matching method called propensity-score matching,
} 
Indian state of Punjab and in Nigeria, households were found to be willing to pay considerably more to connect to piped water systems if they were educated. In Brazil, and the Indian state of Kerala, educated households were found to be more likely to have piped water connections (World Bank Water Demand Research Team 1993). Remarkably, in these World Bank studies, income was not found to be the primary determinant of demand for piped water.

Jalan, Somanathan et al. (2009) used data from the urban subsample of the National Family Health Survey of India in 1998-99 to examine the role of awareness in the demand for water quality. The data indicated that the incidence of diarrhea among young children was quite high at the time, with $16 \%$ of households with children in the 0-3 age group reporting at least one case in the previous two weeks. ${ }^{10}$ Controlling for a number of demographic characteristics Jalan, Somanathan et al. (2009) found that the probability of home water purification, as well as the expected expenditure on purification, increased with years of schooling. This finding concerning the influence of education is striking because of its magnitude. Specifically, the expected expenditure on purification in the top quartile for (female) education is three times higher than the expected expenditure in the bottom quartile, controlling for wealth and other factors. The ratio is the same when one compares the top wealth quartile to the bottom wealth quartile.

It is natural to question whether the schooling variables ${ }^{11}$ are picking up an unmeasured component of income. If income were responsible for the effect of schooling, then since women are much less likely to be in the labor force, ${ }^{12}$ the effect of female schooling should be smaller than that of male schooling. However, in fact, the effect of female schooling is (significantly) larger than that of male schooling. The authors also test for each gender whether the effect of schooling is higher for those individuals in the labor force, and find that it is not.

Using a strategy reminiscent of the work by Ewbank and Preston described above, Jalan et al. (2009) examined occupational differences in the propensity to purify. The probability of

\footnotetext{
${ }^{10}$ This is consistent with fragmentary evidence about the poor quality of water supplies in urban India, with various surveys reporting contamination rates ranging from $10 \%$ of samples in urban Maharashtra to $63 \%$ in the city of Kolkata (McKenzie and Ray, 2004).

${ }^{11}$ The schooling variables used are the schooling in years of the most educated adult man and the most educated adult woman.

12 The probabilities of men and women being employed were 75 percent and 15 percent, respectively.
} 
purification falls as the proportion of adults in the household in occupations not requiring a medical education increases. Interestingly, the fall in the propensity to purify water when the household composition shifts towards workers with a non-medical higher education is about the same as when it shifts towards domestic workers (e.g. maids and nannies), even though the latter are less educated and have lower incomes. But the fall is greater and statistically significant when household composition shifts towards other workers in occupations not requiring a higher education. Why do domestic workers behave like more educated workers? Domestic workers' purification behavior is likely influenced by that of their better educated and higher-income employers.

Further evidence concerning the link between general education and the willingness to pay for water quality comes from a study of the cost of coping with unreliable public water supplies in Kathmandu, Nepal, (Pattanayak, Yang et al. 2005). The authors found that home water treatment costs accounted for somewhat more than a quarter of the total coping costs, other components being collection, pumping, storage, and purchase. The authors did not report the determinants of treatment costs separately, but they found that education is a significant determinant of coping costs, controlling for wages and property values.

\section{Impact of the News Media}

In their study of urban India, Jalan et al (2009) found that media exposure increases the probability of purification, and that the size of the increase depends on the medium. The likelihood of purification rises by 10 percentage points if a female member of the household reads a newspaper at least once a week, by 3.6 percentage points if she listens to the radio at least once a week, and not at all if she watches television at least once a week. This pattern is not particularly surprising since the public sector is still much more dominant in radio broadcasting in India than it is in television, which focuses on entertainment.

The evidence discussed thus far certainly suggests that awareness acquired through education and the news media about water quality as a determinant of risk is an important 
determinant of willingness to pay for safe water. ${ }^{13}$ However, there is no evidence that the measures actually taken by households reduce the risk of diarrhea. There is no correlation in the sample between diarrhea incidence and any of the purification measures taken by households, even boiling or electric filters that sterilize water with ultraviolet radiation. This may be because those households exposed to diseases through means of transmission other than the household water supply are more likely to purify their water and these effects may balance out, on average. Even if the protective measures taken by households are not very effective against the problem they are meant to address, their willingness to pay to address the problem may still be of significance if it makes it more likely that it will be effectively addressed at the societal level through more investment in the water supply and sanitation infrastructure. The link between the demand for environmental quality and government action is discussed in more detail in a subsequent section.

\section{$\underline{\text { Case Studies on Information Provision and Household Behavior }}$}

Jalan and Somanathan (2008) studied a random sample of a thousand households in Gurgaon, a suburb of the Indian capital city. Half of the households were randomly selected and provided with information about their water quality. This group of households is called the "treatment group" while the rest are called the "control group". Gurgaon displayed a high rate of fecal contamination of its water supply, with about $60 \%$ of households' water supply testing positive (i.e., contaminated). Initially, about $40 \%$ of all households were purifying their water at home. Re-visiting the households after two months the researchers found that, conditional on initially not purifying, the frequency of purification had increased by 11 percentage points more among households that had received positive test results (an indicator of contamination) than among control households. ${ }^{14}$

The first thing to note about the effect of this information is that it had a sizeable effect despite a disclaimer provided to the households that the test was not perfectly reliable. Holding

\footnotetext{
13 Jalan et al (2009) show that the expected expenditure on purification (the probability of a purification method being used times its cost summed over all methods) is a lower bound for willingess to pay for safe water.

${ }^{14}$ This was statistically significant at the 5\% level. Expenditure on purification showed a similar pattern. Households that were purifying initially and received a negative test result did not stop purifying at a rate greater than control households (which did not receive test results).
} 
other variables at their means, the estimated effect on the probability of purification of a one standard deviation increase in wealth is also 11 percentage points. The estimated effect of a oneyear increase in schooling of the most educated person in the household is 3 percentage points. Since most purification methods involve some expense, it is not surprising that the result was largely driven by households with above median wealth. The associated expense is an indicator of an increase in households' willingness to pay to protect themselves in response to their increased perception of risk.

The sample used by Jalan and Somanathan (2008) had too few cases of diarrhea for the authors to find an impact on diarrhea risk. But there is some indication that purification did not improve health outcomes as much as might have been hoped. The frequency of contamination, as measured by the test result, was not much lower for purified water stored in the home than it was for unpurified water. In part, this reflects a well-known phenomenon in the public health literature: the tendency for stored water to be re-contaminated. However, it has been found that water contaminated in the home is less harmful than that contaminated outside because household members tend to have acquired immunity to their own germs (VanDerslice and Briscoe 1993).

In the Jalan and Somanathan (2008) study, the impact of information was to raise willingness to pay for safer water. It is not clear if the households' individual efforts took them towards the objective of safer water. But the results do suggest that revealed-preference methods would under-estimate the demand for water quality that households would have if they were fully informed. If households were provided with regular test results for their localities, they would have a better idea about their water quality, and this would presumably raise their willingness to pay to improve it. ${ }^{15}$

An informational intervention that changed the behavior of a much larger fraction of households was carried out in Bangladesh, a country that has been suffering from arsenic contamination of groundwater (Madajewicz, Pfaff et al. 2007). In the district where the

\footnotetext{
${ }^{15}$ The test itself, which could be administered by the government on a sample basis, is not expensive, costing less than $\$ 0.50$ for each kit.
} 
intervention was carried out, 6500 wells were tested for arsenic and were sign-posted as being safe or unsafe depending on whether or not they met the national standard for arsenic concentration in drinking water. Users of the wells were told the concentration in the well they used, whether or not it exceeded the safe limit, and were given general information about the dangers of arsenic. They were exhorted to switch to safe wells.

A follow-up survey of a subsample, conducted six to twelve months after the intervention, collected information on whether people had changed their wells. $60 \%$ of those informed that their well was unsafe changed the well they used, compared to $14 \%$ for those informed that their well was "safe" and $8 \%$ for those in control areas in a different district that did not get information. Treatment and control areas were not randomized; the control was surveyed ex-post. So the difference cannot be directly interpreted as being caused by information. However, control areas are not very different from treatment areas in characteristics such as income, assets and education. So it is implausible that a large part of the differential in the rate of switching to safe wells is due to other factors. In a regression (that excludes control areas) with village fixed effects and household characteristics as controls, the estimated probability of switching to a new well increases by 0.45 when a respondent has an unsafe rather than a safe well. ${ }^{16}$ This is almost exactly the same as the mean difference reported above of 0.46 , suggesting that the controls are uncorrelated to the safety of the well.

When the actual concentration of arsenic was included as an additional control, the effect of the well being safe rather than unsafe dropped by only 0.08 to 0.37 . This suggests that the bulk of the response to the entire package of information received by respondents was related to whether or not the well was reported to be "safe" rather than to the level of arsenic.

The strong behavioral response in the Madajewicz, Pfaff et al. (2007) study is probably due to two or three factors. First, arsenic contamination is a well-known problem in Bangladesh and respondents were probably already aware of the problem in general terms. Arsenic poisoning is known to be cumulative and deadly. The second factor is related to the nature and

\footnotetext{
${ }^{16}$ The inclusion of village fixed effects ensures that only the variation in information between households within the same village is used to infer the effect of information on well switching.
} 
form of the information provided. It was unqualified (there were no disclaimers about the reliability of the test), and households with unsafe wells were urged to switch. Finally, and importantly, the cost of switching wells was low for most households. Most had safe wells within a hundred meters of their homes. It appears that switching to safe wells had an impact on health, as expected. Arsenic levels in urine samples fell for those that changed wells.

Most interventions using information to change behavior do not rely on information alone. For example, in the intervention in Bangladesh, respondents whose wells were considered unsafe were urged to switch to a safe well. If behavioral economics has taught us anything at all, it is that framing matters. In an experiment that considered a new approach for India's Total Sanitation Program in the state of Orissa, India, overt social pressure, in addition to information or education, was applied (Pattanayak, Yang et al. Forthcoming).

In Orissa, one of the poorer states in India, less than $10 \%$ of the population has access to safe water and sanitation. The sanitation campaign focuses on persuading households to construct latrines, with information and education supposed to do most of the job, and subsidies playing a minor role (Pattanayak, Yang et al. Forthcoming). In the experiment, a local community-based organization led "a community walk meant to call attention to poor hygiene, and trigger a collective, emotional response." The organization also supported social sanctions. For example, those who continued with the practice of open defecation were mocked. In addition, households identified by the government as being below the official poverty line were given a substantial subsidy for construction of latrines.

The intervention took place in 20 randomly selected villages with 20 more being monitored as controls. Pattanayak, Yang et al. found that after one year, the intervention was already having a substantial impact. Not surprisingly, the effect was larger for households below the poverty line ( $60 \%$ of all households in the treatment villages), as these households were eligible for the subsidy. Among these households, latrine ownership rose by 34 percentage points. However, the effect was still substantial (a 21 percentage-point increase in latrine ownership) among those households that did not receive a subsidy. Data from the Government 
of Orissa for 2007, one year after the completion of data collection by Pattanayak et al. ${ }^{17}$, showed that 10 of the 20 treatment villages had achieved $100 \%$ coverage.

Before the intervention, over $90 \%$ of households cited open defecation as a cause of diarrhea, indicating a surprisingly high level of awareness of the links between the two. Yet the baseline levels of latrine ownership were very low. This suggests that the campaign may have contributed by changing norms as much as by providing information. If norms are indeed important then we should expect non-linearity in adoption of new behavior in response to information. One aspect of such norms is social approval for following norms and disapproval for not doing so. Even without taking social disapproval into account, however, Boyd and Richerson's (1985) theory of cultural evolution suggests that the behavioral response to information will be non-linear, small at first, and increasing as adoption picks up and changes the culture.

In the Orissa program, the outcome of interest was the probability that a household constructed a latrine in the year following the total sanitation campaign. Dickinson and Pattanayak (undated) estimated this probability as a function of a number of variables, one of them being a "social effect". 18 In one model the "social effect" was simply the percentage of other households in the village that constructed a latrine that year. In the sample of households from the treated villages, that is, the villages exposed to the campaign, Dickinson and Pattanayak (undated) found that the marginal effect of a one-percentage-point increase in the number of households constructing latrines was to raise the probability of construction by $0.84 \%$ (when other variables were held at their means). This is a strong effect but it could be picking up the effects of successful campaigns in particular villages. In a second model, the probability of adoption by a given household was first predicted using only that household's characteristics. In the second stage, adoption was regressed on household and village characteristics and the predicted level of adoption in the village from the first stage. This method produced a very similar result. Now the interpretation that imitation of neighbors was an important determinant of adoption seems much more likely. The only other possible interpretation is that the household-

\footnotetext{
${ }^{17}$ That is, two years after the start of the experiment.

${ }^{18}$ The authors used a probit model.
} 
specific regressors used in the first stage are correlated with some omitted (from the second stage) village-specific characteristic that makes adoption more likely (Manski 1993). This appears less likely than the explanation that culture matters.

To sum up, the evidence that awareness and information are important determinants of the demand for water quality in developing countries is strong. However, this is not to say the phenomenon is universal or independent of context. Jalan and Somanathan's (2008) experiment showed that the effect of information on purification expenditure was wholly driven by those with greater than median wealth. In another study, in the Philippines, 90\% of those asked were aware that beach water was polluted. The stated willingness to pay to improve it was nevertheless extremely low (Choe, Whittington et al. 1996).

\section{Pesticide use}

Pesticide use is another area in which individual action to reduce an environmental hazard is feasible. Most pesticides are dangerous to humans, and farmers trade off health risks against yields. In developing countries, pesticides that are banned or restricted in developed countries are often in widespread use. Hundreds of active ingredients and thousands of formulations are available and actively promoted (Eddleston, Karalliedde et al. 2002). The choice problem for the farmer is quite demanding, and it is easy to see how education could result in a reduction in the use of the more dangerous pesticides without reducing profitability.

The most common method of such education is through the promotion of integrated pest management (IPM) in so-called farmer field schools (Van den Berg and Jiggins 2007). Integrated pest management was originally developed in South-East Asia not out of a concern about the health effects of pesticide use, but as a means of dealing with pest outbreaks brought about by the onset of resistance in pests and elimination of pests' predators. Pesticides are used with much more discrimination in IPM, which relies more on observation-based application and less on prophylactic treatment. A farmer field school consists of regular weekly meetings of a group of farmers with an extension agent over the duration of a crop growing season, usually under a tree in the fields. Small experiments are made and their progress reviewed at the next 
weekly meeting. The method is appropriate due to the complexity of the problem and variations in local conditions.

Van den Berg and Jiggins (2007) reviewed impact evaluation studies of farmer field schools teaching IPM, selecting only studies that met minimum quality criteria. They reviewed three large-sample country-wide studies from Indonesia and Vietnam, two of rice and one of tea, using before-and-after comparisons with the time lag being one year. All three found large declines in insecticide use, exceeding $60 \%$. Secondary data in Indonesia and Vietnam show that insecticide sales per hectare of rice planted increased in these countries over the period of the studies. Thus the decline in insecticide use in the samples was not driven by an overall decline in the countries concerned. One of the studies (Pincus 1999) also examined small control groups of thirty farmers in two provinces in Vietnam. The declines in the control groups were $61 \%$ and $62 \%$ compared to $86 \%$ and $96 \%$ in the treatment groups. In view of the national-level increases, the large declines in the control groups must have been due to local factors. These could have been diffusion from the IPM training programs, information that became available to farmers through other programs and sources, or even (less probably) autonomous responses to pesticiderelated yield declines.

Another six cross-sectional studies, four of them large-scale and all but one related to rice, compared farmers in field schools to those who were not. These six studies found that farmers who had been in the field schools used less insecticide than those who had not by amounts ranging from $35 \%$ to $92 \%$. Yields were also uniformly higher for those farmers who had been to the field schools. These results could be driven by self-selection, since more motivated farmers might join field schools. Nevertheless, the size of the effect makes it seem unlikely that self-selection would explain more than part of the effect. Two of the studies were from Sri Lanka (concerning rice cultivation) and studied farmers who had been to field schools at least five years previously. They found that those farmers still used insecticides at one-third the rate of farmers in the control group. In Sri Lanka, at least, the effect seems quite durable.

Five studies used panel data allowing for difference-in-difference estimation that can control for initial differences in insecticide use between treatment and control groups. Of these, 
four found a significantly greater decline in pesticide use by farmers who had attended field schools, compared to the controls. One study on rice in Indonesia did not find a significant impact on pesticide use. However, van den Berg and Jiggins (2007) point to a re-examination of the data from this study, which showed that three of the four villages from which the controls were drawn were within one kilometer of villages that had farmer field schools. It is, therefore, possible that some diffusion to the control farmers had occurred, especially since eight years had passed since the base year.

Since none of the studies randomized assignment of groups to treatment and control, it is possible that areas chosen for farmer field schools were those more likely to respond. Even with randomization, however, there would be some self-selection of farmers into the field schools, so a full analysis would require tracking farmers who had been offered the chance of being in a farmer field school. These caveats notwithstanding, the evidence does suggest a significant effect on pesticide use.

\section{Links between Awareness and Government Action}

While some important environmental problems are at least partially susceptible to individual action, most are not. Such problems require public action, usually by the government, to correct externalities. This section examines the links and feedback between awareness of environmental risks and government action in developing countries.

\section{Link Between Public Awareness and Demand for Regulation}

An interesting question is whether an exogenous increase in information available to the public, or greater ability to understand such information, would significantly raise the demand for environmental regulation in developing countries. There can be no doubt that rising public awareness of environmental problems has had a major impact on legislation and regulation in developed countries. The publication of the best-seller Silent Spring (Carson 1962) on the toxic effects of chemicals on birds and other organisms in the United States gave an enormous boost to the environmental movement that resulted in much environmental regulation there and in other developed countries (Guha 1999). 
Guha's (1999) global history of environmentalism gives no such clear picture of an exogenous increase in public awareness triggering regulation in developing countries. There are fairly clear instances of an increase in public awareness leading to environmental regulation, but these increases are not obviously linked to external information. For example, in the early 1970's the clear cutting of trees in the Indian Himalayas sparked a protest by villagers concerned about the loss of forests that they needed for agricultural and domestic uses. Most Indian forests are owned and managed by state forest departments. Felling was contracted out. In an incident reported widely in the media, women in a Himalayan village hugged the trees to prevent the contractor's men from cutting them down. The movement against felling by outsiders became known as the Chipko ("stick to") movement. It was one of the influences that led the government of India to pass the Forest Conservation Act in 1980, and in subsequent years, regulations that severely restricted felling in government forests. In this case, there appears to have been no role played by external information.

Environmental regulation around the world has often been triggered by disasters. The world's worst industrial disaster to date, the explosion at Union Carbide's chemical plant in Bhopal, India in December 1984, led to India's first comprehensive environmental protection law, the Environment Protection Act of 1986. This disaster also stimulated legislation in the US that established the Toxic Releases Inventory. In these cases, too, the rise in awareness seems to have been entirely due to the dramatic deterioration in the environment rather than being stimulated by policy measures or research.

However, there seem to have been cases where regulation has itself increased public awareness, leading to pressure on the government to take further measures for environmental protection. One example is the effect of India's Wildlife Protection Act of 1972. This was triggered largely by elite (external and domestic) concerns about the possible extinction of tigers in the wild. The Act passed because Prime Minister Indira Gandhi was in favor of nature conservation and was in a dominant political position (Rangarajan 2001). The establishment of Tiger Reserves led over time to their becoming popular destinations for the upper and middle classes to visit. The annual censuses of tigers in the reserves have figured prominently in the 
media. As a result, a strong middle-class constituency for the protection of tigers has built up over the last few decades. When a 2004 census revealed that one of the reserves had lost all its tigers, probably as a result of poaching, the resulting public outcry forced the government to constitute a Tiger Task Force that included critics of government policy. As a result, a system of independent monitoring of the tiger population has been instituted and external experts have been given a formal role in the making of conservation policy (Anonymous 2005).

\section{Links Between Information, Actions, and Outcomes}

Is there systematic evidence of links between information available to the public, the demand for environmental quality, environmental regulation, and environmental outcomes? Since much of the variance in environmental regulation, or, at any rate, the measurable variance, occurs at the national level, cross-country data are necessary to answer this question.

The first link (i.e., public awareness leading to increased demand for environmental quality) has been examined using data from the third wave of the World Values Survey, carried out in the latter half of the 1990s (Israel and Levinson 2004). The sample included thirty-three countries, seven of them being high-income (OECD) countries, the rest being low and middleincome countries. The survey asked respondents whether they would be willing to pay 20 percent higher prices to protect the environment. Israel and Levinson (2004) found that both increasing income and increasing education raised the probability that respondents would answer "yes" to this question. ${ }^{19}$ Between respondents with no education and those with college education, the difference in the probability was 15 percent. The same pattern was found when the dependent variable was that respondents think that protecting the environment should take priority over economic growth.

Since educated people are more likely to obtain and understand information about environmental problems, this pattern is consistent with what was hypothesized above, that is, that education increases the demand for environmental quality by increasing understanding of environmental hazards. It is not, however, the only interpretation that is consistent with the

\footnotetext{
${ }^{19}$ Israel and Levinson (2004) estimated a linear probability model using country-fixed effects and dummies for 10 income classes and several education classes.
} 
pattern. Inglehart (1995) has proposed that the reason richer and more educated people tend to be more environmentally concerned is that they are more likely to hold "post-materialist" values. This explanation posits endogenous preferences, meaning that consumers' tastes change with the passing of time and increases in income. Inglehart (1995) showed that there is a cohort effect in the data from surveys in several European countries, with later cohorts more likely to hold what he calls post-materialist values, controlling for age. Unlike the information-based explanation, the welfare implications of this interpretation are not straightforward, as is the case for any theory with endogenous preferences.

While the link between information or awareness and demand for environmental quality appears to be consistent with the cross-national data above, there does not appear to be systematic developing-country evidence that bears on the second link in the chain, between the demand for environmental quality and more environmental regulation. Dasgupta, Mody et al. (2001) showed that environmental regulation is strongly correlated with per capita income in cross-country data, but they did not report estimates of the effects of education or awareness levels on regulation. Thus, we do not have evidence on whether increased demand for regulation translates into greater supply. Further investigation of this issue that takes account of political structure, such as whether countries are democracies, would be of interest.

We can, at any rate, directly examine the link between education and environmental outcomes at the country level. Of course, tests of hypotheses using cross-country data are necessarily weak tests. ${ }^{20}$ Torras and Boyce (1998) examined the impact of literacy on various pollution indicators separately for low and high-income countries. ${ }^{21}$ They found that literacy in low-income countries is significantly associated with lower pollution in the cases of sulfur dioxide, heavy particles, dissolved oxygen and percent of people with access to sanitation, but does not have a statistically significant effect in the cases of smoke, fecal coliform, and percent of people with access to safe water. For high-income countries, literacy has the wrong sign in

\footnotetext{
${ }^{20}$ While a correlation not predicted by the theory provides evidence against the theory, a finding consistent with it will usually also be consistent with other theories, because cross-country data allow only a very limited set of controls for possible confounders.

${ }^{21}$ They define the cutoff between low and high income to be $\$ 5000$ at purchasing power parity (PPP). They regressed the various indicators on a cubic function of per capita GDP, and on interactions of a high-income country dummy with the Gini coefficient for income, a measure of civil liberties, and literacy.
} 
two cases, the right sign in two others and is not statistically significant for the other three. Torras and Boyce interpreted literacy as not just an indicator of information that could raise the demand for environmental quality, but as a political variable that would affect the supply of environmental quality through the political process.

Using cross-country data, another paper examined the effect of annual newspaper sales per 1000 people on the percentage of land area in national parks or under similar protection (Bimonte 2002). The sample included middle and high-income European countries, including transition economies. Newspaper sales per 1000 people raised the protected-area-income curve, consistent with the hypothesis that public information raises environmental quality. Finally, another environmental Kuznets Curve study uses the average years of schooling among adults as an education variable, and finds that it raises the levels of carbon dioxide, sulfur dioxide, and nitrogen oxides (Hill and Magnani 2002). ${ }^{22}$

Putting these cross-country results together, the data do not appear to present a clear picture. The theory that public information and awareness result in better environmental quality in developing countries remains an interesting speculation. While the link between public awareness and education and the demand for environmental quality appears to be quite robust, there is no systematic developing-country evidence that this demand translates into increased supply through the political process and government regulation.

\section{Lessons}

The lessons from this review are of two kinds. First, there are substantive implications for environmental policy. It is clear that information provision to the public can be an important tool in addressing some of the major environmental problems in developing countries. Information about hygiene, water quality, and sanitation, when appropriately framed, can induce behavioral changes in households that significantly reduce disease risk. The evidence on the provision of information to farmers on integrated pest management also suggests that this sort of information can result in major reductions in insecticide use. Though not discussed in detail here,

\footnotetext{
${ }^{22}$ The study controlled for a polynomial in per capita GDP, and measures of inequality and trade openness
} 
research over the last two decades on indoor air pollution in developing countries indicates that smoke from cooking fires poses a major health hazard (Ezzati and Kammen 2002). This points to another area where information may be able to play a significant role in encouraging individual actions to improve environmental quality, but additional research and trials are needed.

It would be a mistake to exaggerate the role of information mediated by individual action, however. While changes in hygiene behavior have been shown to significantly reduce disease risk, the levels of risk following such changes in disease-prone environments are still orders of magnitude higher than in developed countries with universal treated water supplies and sanitation. Public action for this and other environmental problems, such as the pesticide problem, cannot be dispensed with if externalities are to be fully addressed.

The other substantive policy-related implication from this review concerns the impact of schooling on the demand for environmental quality. This appears to be of significant magnitude in the case of water quality in urban India. Education also appears to influence stated environmental preferences and willingness to pay worldwide. What remains unknown is the extent to which this demand actually gets translated into action by the state in developing countries. It may be the case that the political processes in developing countries are too insensitive to public opinion and too easily captured by polluter interests to respond in significant ways, stray cases aside. This remains an open question.

The second kind of lesson from this review is methodological and concerns the practice of environmental economics by researchers. Missing information plays an un-ignorable role in the environmental problems of developing countries. Valuation methods that rely on revealed preference run the risk of possibly severe downward bias. ${ }^{23}$ Their use brings to mind the joke used by Stephen Marglin in his graduate microeconomics class at Harvard to highlight the weakness of revealed preference as a welfare valuation tool.

Overheard in the basement of Littauer:

\footnotetext{
${ }^{23}$ Stated preference methods would also be subject to this problem if they did not provide the relevant information. Even if they did, the response may not be immediate, as suggested by the culture hypothesis.
} 
"I took the best way home yesterday."

"How do you know it was best?"

"Because I took it."

In the context of complex environmental problems in which cause and effect are difficult to disentangle without large-sample multi-period studies and randomized trials, it is foolhardy to suppose that individuals capable of observing samples of size one or two will come to the right conclusions and act on them. According to Boyd and Richerson (1985), it was precisely this sampling error that generated the evolutionary selection pressure that led us humans to end up relying heavily on imitation and the associated cultural transmission of beliefs and practices. But when communities have no way to observe and aggregate the relevant information, this cultural conformism can easily lock communities into sub-optimal practices.

Developing countries are not characterized by information-rich environments. This is especially the case for the poorer of their citizens. In this context, caution and careful attention to the information available to people are called for when conducting valuation studies, making welfare judgments, and drawing policy conclusions.

\section{References}

Aiello, A. E. and E. L. Larson (2002). "What is the evidence for a causal link between hygiene and infections?" Lancet Infect Dis 2(2): 103-10.

Anonymous (1992). "Let them eat pollution." The Economist, London, February 8.

Anonymous (2005). Joining the dots: The report of the Tiger Task Force. Report Submitted to the Ministry of Environment and Forests., Government of India.

Begg, C. B. and M. Mazumdar (1994). "Operating Characteristics of a Rank Correlation Test for Publication Bias." Biometrics 50(4): 1088-1101.

Bimonte, S. (2002). "Information access, income distribution, and the Environmental Kuznets Curve." Ecological Economics 41(1): 145-156.

Carson, R. (1962). Silent spring. Boston, Houghton Mifflin.

Choe, K., D. Whittington, et al. (1996). "The Economic Benefits of Surface Water Quality Improvements in Developing Countries: A Case Study of Davao, Philippines." Land Economics 72(4): 519-537.

Cutler, D. and G. Miller (2005). "The role of public health improvements in health advances: the twentieth-century United States." Demography 42(1): 1-22. 
Dasgupta, P. (2004). "Valuing health damages from water pollution in urban Delhi, India: a health production function approach." Environment and Development Economics 9(01): 83-106.

Dasgupta, S., A. Mody, et al. (2001). "Environmental regulation and development: a crosscountry empirical analysis." Oxford Development Studies 29(2): 173-187.

Dickinson, K. and S. K. Pattanayak (undated). Open sky latrines: Social reinforcing in the case of a (very) impure public good, Duke University.

Eddleston, M., L. Karalliedde, et al. (2002). "Pesticide poisoning in the developing world--a minimum pesticides list." The Lancet 360(9340): 1163-1167.

Ewbank, D. C. and S. H. Preston (1990). Personal health behaviour and the decline in infant and child mortality: the United States, 1900-1930. What we know about Health Transition. J. Caldwell. Canberra, Health Transition Centre, Australian National University: 116.

Ezzati, M. and D. M. Kammen (2002). "The health impacts of exposure to indoor air pollution from solid fuels in developing countries: knowledge, gaps, and data needs." Environmental Health Perspectives 110(11): 1057.

Farrell, P. and V. R. Fuchs (1982). "Schooling and Health." Journal of Health Economics 1: 217230.

Fewtrell, L., R. B. Kaufmann, et al. (2005). "Water, sanitation, and hygiene interventions to reduce diarrhoea in less developed countries: a systematic review and meta-analysis." The Lancet Infectious Diseases 5(1): 42-52.

Grossman, M. (1976). The correlation between health and schooling. Household production and consumption. N. E. Terleckyj. New York, Columbia University Press.

Guha, R. (1999). Environmentalism: A Global History. New York, Addison Wesley.

Hill, R. J. and E. Magnani (2002). "An exploration of the conceptual and empirical basis of the environmental Kuznets curve." Australian Economic Papers 41: 239-254.

Inglehart, R. (1995). "Public Support for Environmental Protection: Objective Problems and Subjective Values in 43 Societies." PS: Political Science and Politics 28(1): 57-72.

Israel, D. and A. Levinson (2004). "Willingness to pay for environmental quality: testable empirical implications of the growth and environment literature." Contributions to Economic Analysis \& Policy 3(1): 1254-1254.

Jalan, J. and M. Ravallion (2003). "Does piped water reduce diarrhea for children in rural India?" Journal of Econometrics 112(1): 153-173.

Jalan, J. and E. Somanathan (2008). "The importance of being informed: Experimental evidence on demand for environmental quality." Journal of Development Economics 87(1): 14-28.

Jalan, J., E. Somanathan, et al. (2009). "Awareness and the demand for environmental quality: survey evidence on drinking water in urban India." Environment and Development Economics 14(06): 665-692.

Kenkel, D. S. (1991). "Health behavior, health knowledge, and schooling." Journal of Political Economy: 287-305.

Madajewicz, M., A. Pfaff, et al. (2007). "Can information alone change behavior? Response to arsenic contamination of groundwater in Bangladesh." Journal of Development Economics 84(2): 731-754.

Manski, C. F. (1993). "Identification of endogenous social effects: The reflection problem." The Review of Economic Studies: 531-542.

McConnell, K. E. and M. A. Rosado (2000). "Valuing discrete improvements in drinking water quality through revealed preferences." Water Resources Research 36(6). 
Pattanayak, S. K., J.-C. Yang, et al. (Forthcoming). "Shame or subsidy revisited: Cluster randomized evaluation of social mobilization for sanitation in Orissa, India." Bulletin of the World Health Organization.

Pattanayak, S. K., J. C. Yang, et al. (2005). "Coping with unreliable public water supplies: averting expenditures by households in Kathmandu, Nepal." Water Resources Research 41(2): W02012.

Pincus, J. (1999). The impact of IPM farmer field schools on farmers' cultivation practices in their own fields, FAO programme for Community IPM in Asia.

Preston, S. H. (1996). American Longevity: Past, Present, and Future. Syracuse, NY, Syracuse University: 18.

Rangarajan, M. (2001). India's Wildlife History: An Introduction. Delhi, Permanent Black.

Smith, K. R. (2000). "National burden of disease in India from indoor air pollution." Proceedings of the National Academy of Sciences 97(24): 13286-13293.

Torras, M. and J. K. Boyce (1998). "Income, inequality, and pollution: A reassessment of the environmental Kuznets curve." Ecological Economics 25(2): 147-160.

Van den Berg, H. and J. Jiggins (2007). "Investing in Farmers--The Impacts of Farmer Field Schools in Relation to Integrated Pest Management." World Development 35(4): 663686.

VanDerslice, J. and J. Briscoe (1993). "All coliforms are not created equal: a comparison of the effects of water source and in-house water contamination on infantile diarrheal disease." Water Resources Research 29(7).

WHO (2002). World Health Report 2002: Reducing Risks, Promoting Healthy Life. Geneva, World Health Organization.

World Bank Water Demand Research Team (1993). "The Demand for Water in Rural Areas: Determinants and Policy Implications." The World Bank Research Observer 8(1): 47-70. 